\title{
Functional thyroid gland grown in vitro offers intriguing therapeutic possibility
}

$\mathrm{R}$ esults of a study published in Nature demonstrate that functional thyroid tissue can be generated from embryonic stem cells in culture and that implantation of the tissue can restore a euthyroid state in mice that have undergone radioactive iodine ablation. "By inducing two important genes in stem cells, we were able to push the differentiation of stem cells into thyrocytes, which are responsible for thyroid hormone production in the thyroid gland," says senior investigator Sabine Costagliola of Université Libre Bruxelles. These results open up the possibility of using such a treatment for patients with hypothyroidism.

Congenital hypothyroidism is the most common congenital endocrine disorder, affecting approximately one in 2,000 newborn babies. In adulthood, clinical hypothyroidism is one of the most common endocrine maladies, with a lifelong risk of around 5\%. Additionally, after radioactive thyroid ablation therapy, which is often used as a treatment for thyroid cancers, patients become profoundly hypothyroid and require thyroid-hormone-replacement therapy for the rest of their lives.

In this study, Costagliola and colleagues generated recombinant mouse embryonic stem cells in which expression of Nkx2-1 and Pax8 was induced by doxycycline treatment. Expression of these two transcription factors was sufficient to induce upregulation of known markers of the functional thyroid, including Tshr (thyroid-stimulating hormone receptor), Slc5a5 (sodiumiodide symporter), $\mathrm{Tg}$ (thyroglobulin) and Foxe1 mRNAs. However, the cells rarely formed thyroid-follicle-like structures spontaneously when this approach was used alone.

As Tshr mRNA was upregulated in cells in which expression of Nkx2-1 and Pax8 was induced, the team hypothesized that these cells would be sensitive to TSH. Indeed, when treated with recombinant human TSH, the embryonic stem cells formed 3D structures that were highly similar to thyroid follicles.

The researchers found that the cells in these structures were organized and oriented in a manner conducive to thyroid hormone synthesis. The sodiumiodide symporter was located on the basolateral membrane and thyroglobulin was located intracellularly and in the luminal compartment. Moreover, the team showed that the cells accumulated iodine and that organification of iodine (binding to thyrogloblin) took place within the structures. The process of organification is essential for thyroid hormone synthesis and these results indicate that thyroid hormone was being produced in this cultured tissue.

To test the physiological functionality of the thyroid tissue they had generated in culture, the researchers implanted the tissue into the kidney capsules of mice that had been made profoundly hypothyroid using radioactive iodine ablation. Following receipt of the implant, plasma $\mathrm{T}_{4}$ levels of the mice increased dramatically, with euthyroid levels being achieved in the vast majority of the animals 4 weeks after surgery. Plasma TSH levels were reduced following tissue implantation. Additionally, administration of TSH to these mice increased their $\mathrm{T}_{4}$ levels, indicating that the implanted tissue remains sensitive to TSH. Encouragingly, implantation of the generated thyroid tissue also increased the body temperature of the mice, demonstrating symptomatic rescue of their hypothyroidism.

Progress has been made in the past decade with regards to the treatment of diabetes mellitus, with successful pancreatic transplants being performed and stem cells being used to generate insulin-secreting cells. The results of the present study indicate that regeneration and implantation of another major endocrine tissue could also be possible and that treatment of patients with hypothyroidism could be achieved through the replacement of an entire, functional gland.

"Not only have we produced a tool to permit characterization of the molecular processes associated with embryonic thyroid development," says Costagliola, "but the results of the transplantation also open new avenues for the treatment of thyroid hormone deficiency resulting from congenital hypothyroidism or thyroid ablation therapy in patients suffering from thyroid cancer". The researchers are currently working towards generating functional human thyroid tissue from stem cells derived from human skin using a similar method.

\section{Fiona Mitchell}

\footnotetext{
Original article Antonica, F. et al. Generation of functional thyroid from embryonic stem cells. Nature doi:10.1038/ nature 11525
} 